\title{
LE PRONOM DE NÉGATION: DU LATIN AUX LANGUES ROMANES *
}

\author{
Viviane Cunha*
}

Résumé: Cette brève réflexion sur l'origine de rien dans les langues romanes prétend simplement montrer l'importance de la diachronie dans l'étude des langues et récupérer un peu de la latinité, si méprisée en ces temps de globalisation. L'objectif est avant tout d'ordre pédagogique.

La prédominance, évidente, des études linguistiques synchroniques dans les milieux universitaires actuels reflète un certain manque d'intérêt pour l'étude historique des langues bien que l'étude profonde d'une langue soit inconcevable sans une vision générale de son histoire. Étudier une langue du seul point de vue des résultats démontrés par la synchronie, demeure une vision unilatérale et ne présente aucune approche globale de la langue étudiée. Il convient de rappeler ici que la dichotomie diachronie/synchronie, proposée par Saussure, est une question de méthode, ce qui ne signifie pas qu'il faille privilégier l'une ou l'autre.

\footnotetext{
- Recebido para publicação em janeiro de 2002.

" Professora de Filologia Românica da Faculdade de Letras da UFMG.
} 
La présente étude tend à souligner le rôle de la comparaison dans la synchronie des langues romanes en général et à montrer comment l'analyse d'une variation synchronique de la langue latine peut aboutir à une réflexion pan-romane. J'espère ainsi démontrer qu'une vision plus globale de la latinité reste pertinente dans la pédagogie des langues romanes.

Le XIXème siècle, auquel on doit l'apogée de l'étude historique des langues, peut être considéré comme la période la plus féconde des sciences du langage. Effectivement, les études linguistiques s'y affirment avec leurs propres méthodes et un discours scientifique profondément marqué par les idées du romantisme qui, dans le cadre de la recherche identitaire des peuples européens désireux de valoriser les nationalités, privilégie le Moyen Âge, c'est-à-dire l'époque d'origine des langues et des littératures vernaculaires. Ce siècle se caractérise par le comparatisme; tout ou presque y est objet de comparaison: les mythes, les religions, et surtout les langues indoeuropéennes et leurs branches - soit classiques comme le grec, le latin, le sanskrit et le perse - soit modernes: romane, germanique, slave, celtique, etc. On doit à la méthode comparative l'expansion des études linguistiques dont les postulats sont remarquables pour l'histoire des sciences du langage, puisqu'ils donnent naissance aux diverses grammaires comparées des langues européennes. Comme le remarque le romaniste Vidos (1973: 19), ce qui était une hypothèse de travail dans le domaine de la linguistique européenne, est devenue une réalité scientifique, dans le champ de la linguistique romane.

Selon Jeffers et Lehiste (1989: 17-34), la méthode comparative se fonde sur deux principes primordiaux: l'bypothèse de la parenté linguistique et l'bypotbèse de la régularité. L'hypothèse de la parenté linguistique essaye d'expliquer la similarité des mots dans différentes langues vraisemblablement 
apparentées entre elles, c'est à dire qui se forment à partir d'une proto-langue commune. L'hypothèse de la régularité permet de reconstruire la proto-langue en démontrant que les changements des phonèmes sont réguliers.

C'est en partant de la première hypothèse que nous analyserons l'évolution des pronoms latins, équivalents de rien, dans les langues romanes. Nous essayerons de montrer, ainsi, l'importance de la méthode comparative dans l'étude actuelle des langues romanes, et évidemment, de la latinité, en observant, par exemple, que différents signifiants latins ont abouti à un même signifié dans le monde roman. Cependant, notre intention n'est pas de faire une étude exhaustive, notre objectif principal est d'observer ce qui semble le plus pertinent pour élucider l'évolution du pronom de négation, dans la Romania, et concerne surtout les langues nationales.

Si la latinité est plus qu'une question linguistique, c'est néanmoins la langue qui représente le mieux l'héritage des Romains, puisqu'elle perdure dans les différentes variations diachroniques et diatopiques du latin, tout en conservant une structure qui renvoie à l'origine commune. L'analyse comparée des langues romanes reste donc l'un des instruments les plus efficaces pour une meilleure connaissance de leur proto-langue: le latin vulgaire. J'entends par latin vulgaire la langue parlée dans toutes ses modalités, depuis ses origines les plus lointaines, vers le VIème siècle av. J.C. - époque des premières inscriptions latines - jusqu'au début du roman vers la fin du Vème siècle de notre ère. En effet, après la chute de l'empire romain d'Occident - principale force conservatrice de l'unité linguistique et culturelle de la latinité - commence la fragmentation accélérée de la langue qui va aboutir à la genèse des futures langues romanes.

Les concepts péjoratifs réducteurs utilisés par certains auteurs envers le latin vulgaire tels que: "langue de la plèbe", 
"langue des classes moins favorisées", ou encore, "langue sans observation des normes grammaticales", me semblent peu scientifiques et idéologiques; ils ne sont que la répétition ou la mauvaise traduction de ce qui pour les Romains n'était qu'une terminologie: sermo rusticus, sermo plebeius, etc. Le latin vulgaire, on le sait, est une langue vivante qui présente des variations sociales (sermo honestiores, sermo plebeius, sermo veteranus), des variations historiques ou diachroniques - selon les différentes périodes de la romanisation -, des variations diatopiques ou géographiques (sermo provincialis, sermo ruralis, sermo urbanus) et des variations stylistiques - selon les différents registres -; c'est enfin, la langue parlée par différentes classes sociales (par des gens de professions les plus diverses, d'âges différents, etc.); c'est une langue variée dans le temps et dans l'espace et qui utilise un registre plus ou moins formel ou informel, selon la situation linguistique en question.

Selon Bourciez (1946:119), "pour donner à la phrase [latine] une valeur négative, non était le mot usuel", puisque baut avait disparu dans la langue orale. "Il s'est employé de bonne heure avec d'autres termes contenant déjà une négation: nullus, nemo, nibil, nunquam et vers la fin de l'Empire, contrairement à l'usage classique, des phrases non respondet nibil étaient devenues courantes." Les expressions de type "*non vidi neminem s'est donc établi partout", où se trouvent les modèles des langues romanes nationales: portugais (não tenho visto nenbum); espagnol (non be visto a nadie); ancien français (nul n'ai vëu); italien (non bo vedutto nessuno), roumain (nu vazui pe nimene).

L'une des richesses des langues est dans la capacité qu'elles ont de dire la même chose de plusieurs façons. Le latin vulgaire possède de différentes expressions équivalant à nibil, la forme la plus utilisée dans la langue littéraire. D'après Bourciez 
(1946: 245), cette expression a été remplacée de diverses façons dans la Romania: emploi du neutre pluriel nulla en Italie, en Rhétie et au sud de la Gaule; conservation de rem en Gaule; emploi de *ne gentem en Gaule et en Italie; enfin, emploi de périphrases, en Haute Italie et en Dacie, comme on le verra plus loin. "Les pronoms et adjectifs indéfinis offrent une grande diversité suivant les régions, et le remplacement progressif des termes classiques par des nouvelles combinaisons s'est largement opéré", comme l'a remarqué le romaniste français. (Ibid.: 243)

Parmi les mots que la langue populaire latine consacre comme pronoms indéfinis, figure le vocable rem qui, précédé d'une expression négative ou de la préposition sem, détient la même valeur que l'adjectif verbal nata. Rem, usité au début comme substantif, a commencé à apparaître joint à nata et les deux termes ont pris la place du classique nibil, disparu du latin vulgaire. La langue orale latine présentait au moins quatre équivalents de nibil: rem nata $(m)$; nulla $(m)$ rem; *ne gente; et *ne mica. Les langues romanes ont hérité de toutes ces formes orales.

Le portugais, le galicien et l'espagnol ont gardé le deuxième terme de la forme périphrastique rem nata $(m)$, issue de nulla $(m)$ rem nata $(m)$ 'aucune chose existante', dont: nadla (dans les trois langues) et nadi (ancien espagnol), qui paradoxalement signifiait le contraire dans la langue latine: 'chose née' < res nata. Le gascon a également conservé nat, nade, ce qui pourrait confirmer, par ailleurs, sa spécificité, soit parmi les variantes de la langue d'oc, soit en tant que langue autonome, comme le veulent quelques romanistes. Selon Coutinho (1976: 261), la valeur négative s'expliquerait par la contamination de la négation nulla $(m)$ qui l'accompagnait toujours. Sequeira (1943: 226), ne partage pas cette opinion; selon lui, les mots gardant 
originairement un sens positif, devinrent négatifs par l'influence de l'adverbe non. La locution nulla res nata, en général employée sous la dépendance de non, va subir, petit à petit, une élision, dont il ne restera que le dernier vocable: nata. Selon Bourciez (1946: 245), "(..) les pays ibériques ont favorisé les expressions nemo natus, res nata, qui sont très anciennes", comme on peut le constater chez les auteurs Plaute (cf. Natus nemo in aedibus servat) ${ }^{1}$ et Térence (cf. re nata melius fieri baud potuit)" "de la par ellipse du déterminé un pronom négatif natus, nata (...)."

De nullam rem 'aucune chose', qui avait une valeur affective dans le latin vulgaire, nous avons les formes romanes suivantes: rien (français), ren (occitan), res (catalan), cette dernière forme provenant du nominatif. Remarquons que le catalan adopte, dans ce cas, la même forme que les langues de la Gaule, ce qui réaffirme sa position de langue intermédiaire entre les groupes ibèro-roman et gallo-roman. Le galicien a conservé aussi ren et res, cependant, comme l'affirment les linguistes galiciens, ces expressions "aparecer unicamente con funcion de obxecto directo, en contexto negativo e situados tralo verbo, co mesmo significado ca nada: Non dixo res, Non mercamos ren porque estaban as tendas pechadas" (cf. ALVAREZ et al., 1986: 232).

Selon Sequeira (1943: 227), tout comme rien signifiait 'quelque chose' dans un sens positif, le pronom indéfini aucun, gardait lui aussi une valeur positive. L'auteur cite un exemple du portugais moderne, où la négation prête une valeur négative à l'indéfini: "não tenbo coisa alguma." Selon Sequeira, il s'agit d'un mécanisme psychologique: un vocable qui apparaît en

\footnotetext{
' Plaut., Most. 447. (d'apr. BOURCIEZ, 1946: 245)

2 Tér., Ad, 295. (ibid.)
} 
locution de négation comme substitut d'un mot négatif, devient - par la répétition, négatif lui aussi - même s'il ne renforce pạs l'adverbe de négation. Comme le remarque Sequeira (Ibid.), on doit à la répétition successive le sens négatif du dernier élément des expressions françaises: "je n'ai trouvé nul" et "je n'ai trouvé aucun". D'après Sequeira, dans les phrases du type "Senbor non vi ren"3, extraite de l'ancien portugais, le vocable ren devient un simple renfort de la négation. Et l'on peut remarquer les successives gradations de la perte de la valeur négative de ren, dans les phrases suivantes: Nom valen rem, ${ }^{4}$ Nom fazia rem pelo corpo ${ }^{5}$ Nom jouve en terra ren ${ }^{\sigma}$ (extraites de la prose médiévale portugaise). Néanmoins, dans la poésie médiévale galicienne portugaise on trouve très fréquemment la forme nulba ren signifiant négation, soit en raison de la présence de la forme négative nulba, soit par influence de la poésie occitane, comme on peut le constater dans l'extrait de la poésie du XIIIème siècle d'Alphonse X le Sage: "Se[n] nulba ren, / sen vosso ben, / que tanto ei deseiado."

D'autre part, les langues de la Romania Orientale - l'italien et le roumain - ont pris, respectivement, les périphrases *ne gente 'aucune personne' et *ne mica 'ni une miette', comme on peut le constater dans les formes modernes de l'italien niente et du roumain nimic, nimica. Le français a également hérité de l'expression ne gente, mais celle-ci n'a pas la même valeur que rien; il s'agit de la forme substantivée néant. Selon Bourciez (1946: 245), dans l'ancien français on retrouve la forme noient,

\footnotetext{
${ }^{3}$ Rei Artur, d'apr. Sequeira (1946: 235).

'Nob. do Conde D.Pedro, ibid.

${ }^{5}$ Ibid.

"Rei Artur, ibid.

${ }^{7}$ CV 362, cit. d'après Spina (1991: 287).
} 
de même origine. Ces formes résultent de la négation renforcée.

D'après Sequeira (1943: 230), il y avait, au Moyen Âge, l'habitude d'intensifier la négation, phénomène qui n'est pas une exclusivité des langues romanes, mais existe aussi dans toutes les langues occidentales. Selon Bourciez, cette tendance remonte au latin vulgaire, comme on peut le vérifier dans le passage suivant: "Une habitude qui, dans la langue vulgaire, date déjà des Comiques, consistait à renforcer la négation par des termes désignant de très petits objets. Ainsi chez Plaute (...) "non nauci facere" (Most. 404) et chez Térence "non flocci pendere" (Eun. 411)." 8

Selon Sequeira (Ibid.), dans l'ancien français, on trouve des expressions: je ne bois pas gote 'ni une goutte', tu ne mangeras mie 'pas même une miette'. L'ancien italien se servait de divers vocables pour renforcer la négation, et certains sont encore usités dans la langue moderne, comme: punto, affato, mica, dont nous avons l'exemple suivant: "Non è punto vero" (extrait de Sequeira). L'espagnol utilisait des mots qui désignaient de petites choses: un grano, un figo, un bledo. Toutes ces expressions servaient à renforcer la négation et les langues romanes les ont conservées jusqu'à nos jours.

Pour renforcer la négation, l'ancien portugais gardait, à côté de nada, un autre pronom à valeur d'indéfini. Il s'agit de l'expression migalba < micacula (diminutif de mica), précédée de la particule nem, d'où: nem-migalha et par dissimilation nemigalha ou namigalha (d'après NUNES, 1975: 267). Un échantillon se trouve, parmi d'autres, dans le Livro de Linhagens: "Esta dona Marinha non falava nemigalba". "

\footnotetext{
${ }^{8} \mathrm{Cf}$. Bourciez (1946: 119).

${ }^{9}$ Cf. Nunes, Crest. Arc. éd. 1981.
} 
Chez Gil Vicente - un écrivain intermédiaire entre le portugais ancien et moderne - on trouve encore la forme nemigalba 'ni une miette', comme dans l'exemple suivant: "Näo revolvais arama, / Que näo trago nemigalba". ${ }^{10}$

D'après Sequeira, le portugais fit aussi un large usage des termes: ponto, parte, gota, passo, ren, parallèlement aux autres langues romanes. Et selon lui, le processus d'évolution, en français comme en portugais est le même pour ces mots.

En ce qui concerne l'adjectif latin nullus, celui-ci est passé dans les langues romanes, où il appartient à la classe des adjectifs, ou à celle des pronoms indéfinis, ou encore, à celle des substantifs. En effet, selon Bourciez (1946: 245), "L'indéfini négatif nullus est resté presque partout," tandis que nemo ne se conservait que dans la Romania Orientale: nime (roumain), nimo (ancien italien), nemos (logoudorien), nimmu (corse). La recomposition nec ullus n'apparaît qu'au nord de la Gaule (cf. l'ancien français neül). Pourtant, les composés par unus sont très répandus: nec unus se trouve dans la Peregrinatio ad Loca Sancta (cf. ut nec unam babitationem habeat) ${ }^{11}$. De la même origine nous avons les formes romanes suivantes: niuno (italien), negu (occitan), nenbum (portugais), ninguno (espagnol), ningu (catalan) et nici-un (roumain). ${ }^{12}$

"Comme terme négatif, nullus tient le premier rang. Mais à coté il se produit des composés tardifs de unus; nemo n'est plus usité partout, et nibil sort peu à peu d'usage (peut-être le retrouve-t-on cependant dans le prov. mod. niéu 'nullement'." (cf. BOURCIEZ, ibid.: 97) De la forme du neutre pluriel nulla

\footnotetext{
${ }^{10}$ Auto da Feira, cit.d'apr. Sequeira (1943: 234).

"Per., 8, I.

12 D'après Bourciez, ibid.
} 
nous avons les formes du frioulain nuye et du romanche nola, en ce qui concerne la Rhétie.

Selon Bec (1970: 410), les formes occitanes $\boldsymbol{n u l l}$ (masc.) et nulla (fém.) sont originaires du latin nullus, et les variantes phonétiques palatalisées nulb / nulba et encore: lunb / lunba (par métathèse) sont issues de la forme vulgaire *nullius. Le romaniste français le signale comme pronom équivalant à $n u l$ (semblable en occitan et en français).

D'après Fernandez Gonzalez (1985: 310), "El primer problema que plantean los indefinidos es que no forman una categoria netamente delimitada, ni tienen una organizacion interna como las que tienen el sustantivo, el posesivo o el verbo en provenzal antiguo." Selon le philologue espagnol, on trouve dans l'ancien occitan (ou provençal) les formes nul, nulb < nullus, soit comme pronom, soit comme adjectif.

Nous ne traiterons pas ici des autres fonctions qu'occupe nullus dans le cadre de la grammaire des langues romanes, car cela dépasse l'objectif de notre étude.

Les exemples collationnés ci-dessus donnent une idée de la diversité des signifiants pour un même signifié de négation dans les langues romanes, soit par allotropie, soit par changement sémantique, en raison des variations du latin vulgaire, et nous permettent de soutenir avec Meillet que la méthode comparative, même si elle ne peut pas expliquer toute la complexité de la langue parlée par les peuples latins et latinisés dans l'antiquité romaine, reste un instrument très efficace quand il s'agit de tracer l'histoire des langues romanes. (cf. MEILLET, 1970: 7). 
Resumo: Este breve questionamento sobre a origem do pronome nada - nas línguas românicas - pretende simplesmente mostrar a importância da diacronia para os estudos das línguas, e de certa forma, resgatar um pouco da latinidade, tão menosprezada nesses tempos de globalização. O objetivo é, principalmente, de ordem pedagógica.

\section{Références bibliographiques}

ALVAREZ, R.; MONTEAGUDO, H.; REGUEIRA, X.L. Gramatica galega. Vigo: Editorial Galaxia, 1986.

BEC, Pierre. Manuel pratique de philologie romane. Paris:Picard, 1970/1971. (2 vol.)

BOURCIEZ, E. Éléments de linguistique romane. Paris: Klincksieck, 1946.

COUTINHO, Ismael de Lima. Pontos de gramática bistórica. Rio de Janeiro: Ao Livro Técnico, 1981.

FERNANDEZ GONZALEZ, J. R. Gramática bistórica provenzal.Oviedo: Universidad de Oviedo: Servicio de Publicaciones, 1985.

JEFFERS, Robert J.; LEHISTE, Ilse. Principles and Methods for Historical Linguistics. Cambridge: Mass. and London, Engl., The MIT Press, 1989. 4th Printing.

MEILLET, A. La méthode comparative en linguistique bistorique. Paris: Honoré Champion, 1970.

NUNES, J.J. Compêndio de gramática bistórica portuguesa. Lisboa: Livraria Clássica Editora, 1975.

NUNES, J.J. Crestomatia arcaica. Lisboa: Livraria Clássica Editora, 1981. 
SEQUEIRA, F.J. Martins, Aspectos do português arcaico. Lisboa: União Gráfica, 1943.

SPINA, Segismundo. A lírica trovadoresca. São Paulo: Edusp, 1991. VIDOS, B.E. Manual de linguística románica. Madrid: Aguillar, 1973. 